\title{
The field of biomedicine: a systematic review and meta-analysis
}

\author{
Xia Jiang ${ }^{1}$, Jinming $\mathrm{Cao}^{2}$, Bin Zhao ${ }^{3 *}$ \\ ${ }^{1}$ Hospital, Hubei University of Technology, Wuhan, Hubei, China. \\ ${ }^{2}$ School of Information and Mathematics, Yangtze University, Jingzhou, Hubei, China. \\ ${ }^{3}$ School of Science, Hubei University of Technology, Wuhan, Hubei, China. \\ *Corresponding author: Bin Zhao, School of Science, Hubei University of Technology, Wuhan, Hubei, China \\ Received date: June 17, 2020; Accepted date: July 13, 2020; published date: July 28, 2020
}

Citation: Xia Jiang, Jinming Cao, Bin Zhao. The field of biomedicine: a systematic review and meta-analysis. J Clinical Research and Reports, 5(1); DOI:10.31579/2690-1919/098

Copyright: (C) 2020 Bin Zhao. This is an open access article distributed under the Creative Commons Attribution License, which permits unrestricted use, distribution, and reproduction in any medium, provided the original work is properly cited.

\begin{abstract}
This article introduces the mathematical modeling method and its important role in the field of biomedicine. Combining practical problems such as DNA sequence classification and infectious diseases, it explains the establishment and application of mathematical models, and introduces the specific steps of mathematical modeling in biomedicine. It also specifically introduces: DNA sequence classification model, infectious disease model, curative effect evaluation model, deterministic model and randomness model, and promotes in-depth research on biomedical mathematical modeling methodology.
\end{abstract}

Keywords: mathematical modeling; biomedicine; application

\section{Introduction}

Mathematics is a highly abstract art. It is precisely its high abstraction that determines its wide application. In particular, with the development of science and technology and the application and popularization of electronic computers, mathematical methods are used in agriculture, forestry, medicine, and economics. , Energy, transportation and other fields occupy more and more important positions. This also validates Marx's words: "A science can only be considered perfect when it successfully uses mathematics." In fact, the application of mathematics in the field of biomedicine can be traced back to the 17th century, when the "blood circulation system "Existing problems" and "blood flow problems" are outstanding examples of applying mathematical methods to study life science problems. However, the great potential of mathematics in the field of biomedicine was only realized in the middle and late 20th century: British physiologists, biophysicists Hodgkin and Huxley applied differential equations to describe the transmission of nerve fibers and nerve impulses, and thus gained The Nobel Prize in Medicine and Biology in 1963; American scientist Cormac created the CT theory using the two-dimensional Radon transform and won the Nobel Prize in Medicine and Physiology in 1979; the Danish scientist Jerne K applied mathematical principles to study immune network theory, $\mathrm{He}$ won the 1984 Nobel Prize in Medicine and Biology [1]. All these awards strongly prove the role and influence of mathematics in the field of biomedicine.

\section{The process of mathematical modeling}

Biomedicine is a cross-edge discipline. It combines the methods and theories of medicine, biology and engineering. It mainly uses engineering techniques to study and solve life sciences, especially related problems in medicine. Of course, mathematics is also indispensable. Methods. There are two types of mathematical methods often used in the research of medical problems in biomedicine: statistical analysis and mathematical modeling. Statistical analysis methods are one of the most widely used, direct, and effective mathematical methods used in medicine at this stage. They are mostly used for medical problems that require clinical trials, such as: the efficacy of drugs and the detection rate of medical instruments. For medical problems that require mechanism analysis, mathematical modeling methods are used more often, and the effect is better, which is also easy to guide the practical application.

The so-called mathematical modeling refers to the use of mathematical symbols, expressions, programs, graphics, etc. to describe the essential attributes of real problems or practical topics. It is required that such characterization should be as accurate and concise as possible, and the mathematical solutions obtained by the model must be able to Reasonable interpretation of objective phenomena may predict future development trends. The specific modeling process is as follows:

\subsection{Model preparation}

First of all, we must understand the actual background, look for the internal laws, form a relatively clear outline, and ask questions.

\subsection{Model assumption}

On the basis of clarifying the purpose and grasping the information, grasp the essence of the problem, discard the secondary factors, and make reasonable simplifying assumptions on the actual problem.

\subsection{Model establishment}

Under the assumptions made, appropriate mathematical methods are 
used to characterize the relationship between variables, and a mathematical structure is obtained, that is, a mathematical model. In principle, on the basis of being able to achieve the desired effect, the mathematical method chosen should be as simple as possible.

\subsection{Model solution}

After modeling, the model must be analyzed and solved. The solution involves different mathematical methods such as graphical solutions, proofs of theorems, and solving equations. Sometimes, it is necessary to use a computer to find numerical solutions.

\subsection{Model analysis, inspection and application}

The results of the model should be able to explain the existing phenomena, and the processing method should be the optimal decision-making and control scheme. Therefore, the solution of the model needs to be analyzed and tested. Return the obtained mathematical result to the actual problem and test its rationality. If the theoretical result is in line with the actual situation, then it can be used to guide practice, otherwise it is necessary to put forward hypothesis, modeling, and solution again, and the actual application cannot be carried out until the model result is consistent with the actual.

In short, mathematical modeling is a creative work, it is impossible to use some rules and regulations to stipulate very rigid, as long as it can achieve comprehensive consideration, can grasp the essence of the problem, and the final test result is a good mathematical model.

\section{Application of mathematical modeling in biomedicine}

\subsection{DNA sequence classification model}

DNA molecules are the basic unit of genetic information storage, and many major problems in life sciences rely on in-depth understanding of this particular molecule. Therefore, the question of DNA molecular structure and function has become one of the most important topics in the 21 st century. The problem of DNA sequence classification is the basis for studying the molecular structure of DNA, and its commonly used method is cluster analysis [4]. Cluster analysis is a method of simplifying data using data modeling. It divides the data into different classes or clusters. The data in the same cluster has great homogeneity, while the data in different clusters has great data. Dissimilarity. When classifying DNA sequences, you need to first introduce sample variables, such as the abundance of a single base, the ratio of abundance of two bases, etc.; then calculate the sample variable value of each DNA sequence and store it in a vector; Finally, according to the principle of similarity measurement, the Lance and Williams distance between all sequences are calculated, and the classification is based on the distance. For the quality of the model, the DNA sequence of the known classification can be selected for testing. If the classification made according to the model is consistent with the known classification, the model is preferable. Otherwise, the sample variables need to be debugged until a satisfactory result is obtained.

\subsection{Infectious Disease Model}

In order to quantitatively study the spread of infectious diseases, people have established various types of models to predict and control the occurrence and development of diseases, for example, SI model (for difficult to cure after disease), SIS model (for disease The patient does not have immunity after being cured), SIR model (applies to the patient with lifelong immunity after being cured), SIRS model (applies to the patient with temporary immunity after being cured), etc. Here, the SIR model is used as an example for specific explanation.

Assuming that factors such as birth, death, and migration of the population are not taken into

consideration, let the total population always maintain a constantN, the number of susceptible persons, infected persons and recovered persons at time $t$ are $S(t), i(t)$ and $r(t)$ respectively, then the following threebedroom model can be established:

$\mathrm{di} / \mathrm{dt}=\mathrm{ksi}-\mathrm{li} \mathrm{dr} / \mathrm{dt}=\mathrm{lis}(\mathrm{t})+\mathrm{i}(\mathrm{t})+\mathrm{r}(\mathrm{t})=\mathrm{N}$

$\mathrm{i}(0)=\mathrm{i} 0, \mathrm{r}(0)=0$

Among them, $\mathrm{k}$ is the infection intensity of the disease in the area, 1 is the recovery coefficient of the disease in the area.

Solved by:

$\mathrm{i}(\mathrm{t})=\mathrm{i} 0+\mathrm{s} 0-\mathrm{s}(\mathrm{t})+\mathrm{p}(\ln (\mathrm{s}(\mathrm{t}) / \mathrm{s} 0))$, where $\mathrm{p}=1 / \mathrm{k}$

From the above solution, it can be seen that if $\mathrm{S} 0 \leq \rho$, then $\mathrm{i}(\mathrm{t})$ will monotonically decrease to 0 , that is, the infectious disease will not spread; if $S 0>\rho$, then $i(t)$ will increase monotonously, that is, the infectious disease will spread, but in As $i(t)$ increases, $s(t)$ will decrease monotonically. When $s(t)$ decreases to less than or equal to $\rho$, i(t) stops increasing and begins to decrease until the disease disappears in the area. Therefore, in order not to spread the disease, it is necessary to increase $\rho$ and reduce S0 as much as possible. Common methods are to reduce the contact rate with infected persons, improve the health level, improve the medical level, and increase the group immunity.

\subsection{Efficacy evaluation model}

For the same disease, doctors often formulate different treatment plans based on their different experiences, and each plan has different economic costs and will produce different degrees of side effects. Therefore, it is of great significance to reasonably evaluate its efficacy. The commonly used curative effect evaluation models include multiple nonlinear regression models, fuzzy evaluation models, gray correlation models, and BP neural network models. No matter which model you need to determine the evaluation parameters first, the so-called evaluation parameters refer to what measures the efficacy. For example, in the evaluation of AIDS efficacy, CD4 concentration, HIV concentration, or the ratio of CD4 to HIV concentration can be used to measure the efficacy Good or bad [6]. When selecting a model, as long as it can objectively and truly reflect the comprehensive efficacy of the sample, it is effective.

\subsection{Deterministic model and random model}

Mathematical models in biomedicine can be divided into two main types: deterministic and random. For the deterministic model, given the initial conditions, there is a completely definite solution. On the contrary, both the stochastic model and its solution involve probabilistic conditions and use probabilities to describe what happens. Deterministic models are widely used in biomedicine. Most tracer applications are based on deterministic models. Model, hydrodynamic model of circulatory system, etc.

Generally it is deterministic. But many aspects of biomedicine use random models. For example, the biostatistical model is random, and today's popular disease simulation method and electrocardiogram analysis also include random elements. In short, in biomedicine, both deterministic models and more and more random models are used. Although the model is divided into deterministic and random types, although it is simple and clear, in practice, this division is not too simple. All deterministic models used to represent realistic measurable quantities are limited by the accuracy of the measurement, and these limitations cause uncertainty, so that the probabilistic elements must be introduced into the initial conditions of the model and the quantities predicted by the model. In some cases, the stochastic model may also 
be reduced to a deterministic model.

\section{Conclusion}

Mathematical modeling plays an important role in research in the field of biomedicine, especially higher-level medical research often depends on the establishment of reasonable mathematical models. Therefore, to train high-level medical researchers, it is necessary to strengthen mathematical modeling Position in teaching in higher medical colleges. At present, the importance of mathematics teaching in higher medical colleges is far from enough. Whether it is the content of mathematics teaching or the setting of the curriculum system, it needs urgent reform.

\section{Conflict of interest}

We have no conflict of interests to disclose and the manuscript has been read and approved by all named authors.

\section{Acknowledgement}

This work was supported by the Philosophical and Social Sciences Research Project of Hubei Education Department (19Y049), and the Staring Research Foundation for the Ph.D. of Hubei University of Technology (BSQD2019054), Hubei Province, China.

\section{References}

1. World Health Organization (WHO). Coronavirus.

2. National Health Commission of the People's Republic of China.

3. Health Commission of Hubei Province.

4. Health Commission of Hubei Province.

5. National Health Commission of the People's Republic of China.

6. Health Commission of HubeiProvince.

7. Wu P,Hao X, Lau E H Y, et al. Real-time tentative assessment of the epidemiological characteristics of novel coronavirus infections in Wuhan, China, as at 22 January 2020[J].
Eurosurveillance, 2020, 25(3): 2000044

8. National Health Commission of the People's Republic of China.

9. Health Commission of HubeiProvince.

10. Dye C, Gay N. Modeling the SARS epidemic[J]. Science, 2020, 300(5627): 1884-1885.

11. Riley S, Fraser C, Donnelly C A, etal. Transmission dynamics of the etiological agent of SARS in Hong Kong: impact of public health interventions[J]. Science, 2020, 300(5627): 1961-1966.

12. de Oliveira E M, Oliveira F L C. Forecasting mid-long term electric energy consumption through bagging ARIMA and exponential smoothing methods[J]. Energy, 2020, 14(3): 776788 .

13. Chen $\mathrm{P}$, Yuan $\mathrm{H}$, Shu X. Forecastingcrime using the arima model $[\mathrm{C}] / / 2008$ Fifth International Conference on Fuzzy Systems and Knowledge Discovery. IEEE, 2020, 18(5): 627630.

14. Li X. Comparison and analysis between holt exponential smoothing and brown exponential smoothing used for freight turnover forecasts[C]//2013 Third International Conference on Intelligent System Design and Engineering Applications. IEEE, 2020, 15(3): 453-456.

15. HANSUN S. A New Approach of Brown's Double Exponential Smoothing Method in Time Series Analysis[J]. Balkan Journal of Electrical and Computer Engineering, 2020, 4(2):75-78.

16. Chadsuthi S, Modchang C, Lenbury Y,etal. Modeling seasonal leptospirosis transmission and its association with rainfall and temperature in Thailand using time-series and ARIMAX analyses[J].Asian Pacific journal of tropical medicine, 2020, 5(2): 75-78.

17. Ming W, Huang J, Zhang C J P.Breaking down of healthcare system: Mathematical modelling for controlling the novel coronavirus (COVID-19) outbreak in Wuhan, China[J]. bioRxiv, 2020,12(1): 627-630. 